\title{
Vaccins \\ et professions \\ exposées
}

Dominique Gendrel

> Les vaccinations pour des risques professionnels sont depuis longtemps prises en compte et précisées aussi bien dans le code du travail que dans les recommandations vaccinales et concernent une grande diversité d'activités. Les professionnels de la santé sont en première ligne. Le but des vaccinations est double. II est à la fois de protéger le soignant du risque de contracter une maladie pendant son travail, mais aussi de l'empêcher de transmettre la maladie à des patients fragilisés. II est important d'être attentif aux recommandations, et il faut réfléchir à la façon de les étendre, en particulier aux enseignants et personnels des écoles maternelles et primaires, pour ce qui concerne en particulier la coqueluche, la varicelle, la rougeole et l'hépatite $A$. Une autre population cible, qui est trop peu prise en compte, est celle des étudiants en médecine, souvent insuffisamment protégée et insuffisamment avertie. <

Protéger par la vaccination le sujet exposé à des risques professionnels est depuis longtemps pris en compte par les différentes législations [1]. Mais la diversité des situations fait que les recommandations sont souvent dispersées dans des textes réglementaires de différentes origines. En France, par exemple, des textes abordant la question relèvent du code du travail, d'autres du code de la santé publique, certaines recommandations sont propres à certaines professions, sans oublier les recommandations sanitaires dans les armées [2]. Dans la plupart des pays, les vaccinations pour prévenir un risque professionnel (professions de santé ou autres) sont recommandées mais ne sont pas obligatoires. II suffit de consulter pour cela l'édition francophone du guide canadien d'immunisation qui est très claire sur le sujet [3]. La situation est plus compliquée en France, certaines vaccinations pouvant être obligatoires pour exercer une profession, d'autre seulement recommandées.

Article reçu le 20 février 2007, accepté le 5 mars 2007.

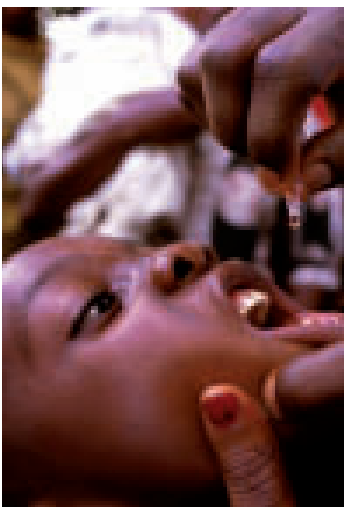

Service de Pédiatrie, Hôpital CochinSaint Vincent de Paul, 24, rue du Faubourg Saint-Jacques, 75674 Paris Cedex 14, France. dominique.gendrel@ svp.ap-hop-paris.fr

\section{Définir des groupes à risques}

Le but de la vaccination des professions exposées est double. II s'agit tout d'abord de protéger d'un risque infectieux les membres d'une profession exposée, mais également d'empêcher les professionnels eux-mêmes de transmettre certaines infections à des personnes dont ils ont la charge. Ce deuxième objectif est particulièrement adapté aux professions de santé, mais s'applique également aux professionnels s'occupant de collectivités d'enfants. Ce terme de collectivités d'enfants, présent dans toutes les recommandations françaises $[1,3]$, peut être interprété de façon large et permet de proposer des vaccinations utiles à de nombreux sujets. Les personnels de crèches et d'institutions vouées à l'enfance et à la jeunesse handicapées sont clairement précisés dans les textes. II faut réfléchir à l'extension des recommandations vaccinales à tout le personnel enseignant s'occupant d'enfants, au moins en ce qui concerne la rougeole, la varicelle, la coqueluche, l'hépatite A et la grippe.

Une population qui doit être particulièrement ciblée est celle des étudiants et élèves en formation, en particulier dans les professions de santé. Dans les écoles d'infirmières ou de sages-femmes, les obligations et recommandations vaccinales sont clairement exprimées lors de l'entrée dans la formation et bien appliquées. En effet, elles sont régies par le code du travail, qui donne des informations précises, et par le code de la santé publique. En revanche, les étudiants en médecine sont les parents pauvres de cette réglementation. Nous n'in- 
sisterons pas ici sur les insuffisances de la médecine universitaire, mais il est important de sensibiliser les étudiants au début de leurs études de médecine, et avant qu'ils ne fréquentent l'hôpital, aux risques encourus et de leur exposer les recommandations et obligations vaccinales.

Les professions concernées sont particulièrement nombreuses et diverses. Elles sont précisées tous les ans dans le Bulletin Épidémiologique Hebdomadaire, dans un fascicule consacré au calendrier vaccinal et qui inclut les recommandations particulières aux professions exposées [1]. Un fascicule est également consacré aux recommandations (incluant les vaccinations) destinées aux voyageurs [4]. Par ailleurs, l'édition 2006 du Guide des Vaccinations est en ligne sur le site Internet du Ministère de la Santé (www.sante.gouv.fr). Il est particulièrement utile et très complet, et permet d'obtenir la plupart des renseignements souhaités concernant les vaccinations des personnels exposés [2]. La plupart des recommandations, dont les plus complexes, concernent les personnels de santé et les élèves se destinant à une profession de santé en formation dans différentes facultés ou écoles.

Certaines professions sont exposées à une maladie invalidante ou empêchant la poursuite de l'activité. Celles-ci sont rappelées dans le Tableau I, directement issu du Bulletin Épidémiologique Hebdomadaire 2006 [1].

\section{Identification des professions à risques particuliers}

La liste de ces professions est publiée chaque année dans les recommandations vaccinales émises par la Direction de la Santé (Bulletin Épidémiologique Hebdomadaire). Ces recommandations sont mises à jour par le Comité Technique de Vaccinations et par le Conseil Supérieur d'Hygiène Publique de France. Cependant, elles peuvent être étendues. Dans le cas de la vaccination contre la grippe par exemple, les recomman- dations s'adressent au personnel navigant des bateaux de croisière et des avions, et au personnel des industries du voyage accompagnant les groupes de voyageurs. Mais ces recommandations sont souvent ignorées des accompagnants bénévoles ou de ceux qui exercent une activité de ce type de façon épisodique. II en est de même pour la vaccination contre l'hépatite $A$. Les professions exposées sont parfois mal connues et l'obligation vaccinale n'est pas satisfaite si les personnes ne dépendent pas d'une administration importante dont les services de médecine du travail sont bien organisés. C'est vrai en particulier pour les personnels temporaires ou bénévoles de crèches familiales ou associatives. Le risque professionnel est souvent mal pris en compte, et c'est au médecin traitant de conseiller son patient.

Un exemple particulièrement illustratif est celui de la vaccination contre la leptospirose ${ }^{1}$. Dans les grandes entreprises ou instituts s'occupant de voierie ou dans les mairies très importantes au sein desquelles les systèmes de contrôle existent, les médecins du travail sont très avertis des types de métier et peuvent apprécier les risques, et conseiller ou non une vaccination contre la leptospirose. Mais pour un ouvrier agricole qui va s'occuper

${ }^{1}$ La leptospirose est une zoonose largement répandue dans le monde, due à la bactérie Leptospira interrogans. Ses principaux réservoirs sont les rongeurs, en particulier les rats, qui excrètent la bactérie dans les urines. Les leptospires se maintiennent assez facilement dans le milieu extérieur (eau douce, sols boueux), ce qui favorise la contamination. La maladie chez l'homme, bien que souvent bénigne, peut cependant être sévère, pouvant conduire à l'insuffisance rénale, voire à la mort dans 3 à $5 \%$ des cas.

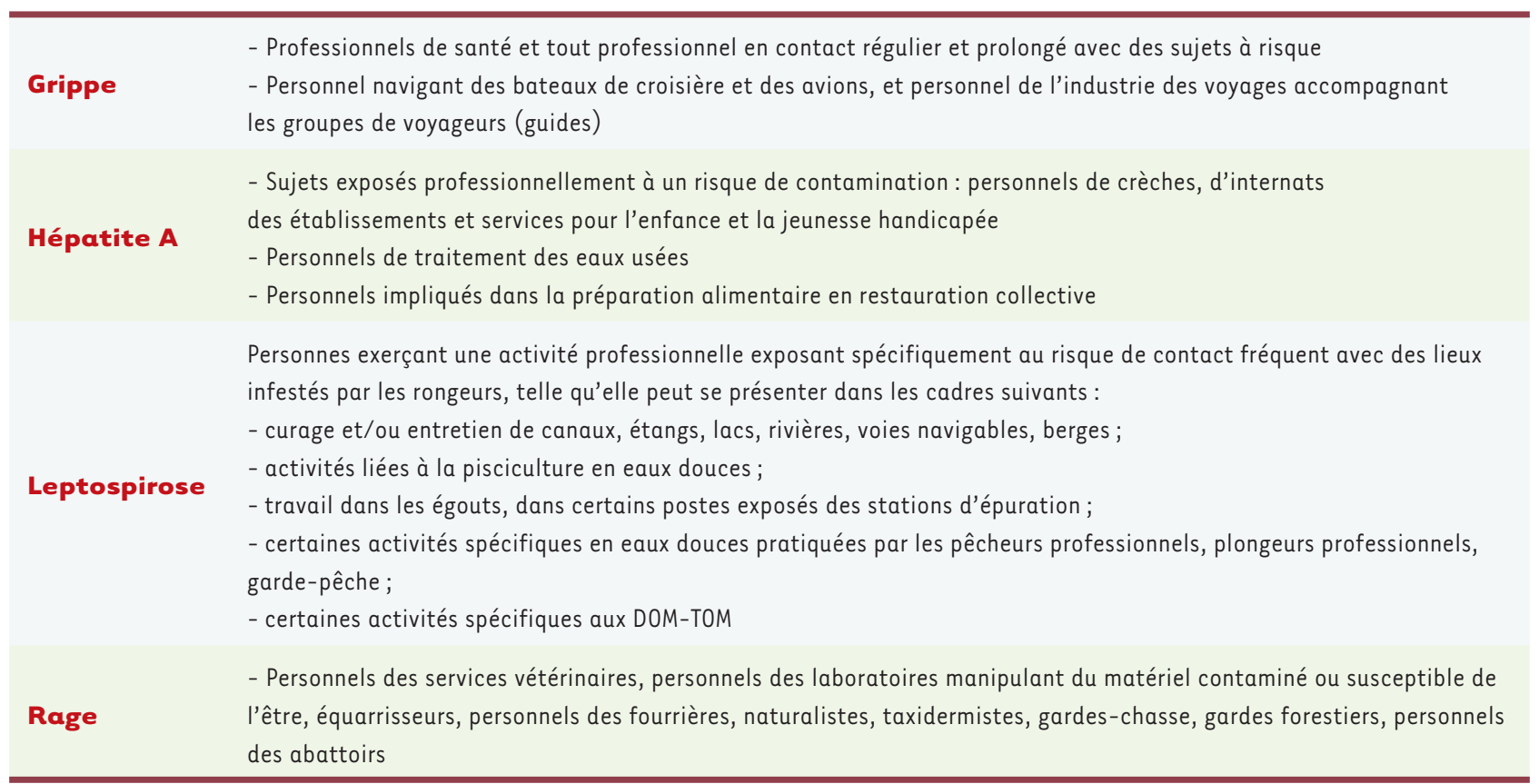

Tableau I. Recommandations particulières des vaccinations pour certaines professions (d'après le calendrier vaccinal publié dans le Bulletin Épidémiologique Hebdomadaire [1]). 
de temps en temps du curage d'un étang ou d'un nettoyage de berges, le risque est plus difficile à évaluer, et il n'y a souvent pas d'organisation de médecine du travail importante et suffisante pour donner ces conseils, et c'est au médecin traitant qu'incombera cette mission. Cette attitude a été rappelée récemment en 2005 dans un avis du conseil Supérieur d'Hygiène de France [1]. Compte tenu du faible nombre de leptospiroses en métropole, surtout celles dues au sérotype icterohemorragia, et de la complexité du protocole vaccinal, la vaccination ne sera proposée qu'en cas de risque important et avéré que les mesures individuelles de protection ne suffisent pas à couvrir. Le médecin du travail doit décider ou non de la vaccination des professions exposées, mais il n'y a pas lieu de vacciner pour des activités récréatives. L'attitude peut être différente dans les DOM-TOM où l'incidence de la leptospirose est plus importante. Quant à la vaccination contre la rage des personnels concernés en France, en général il y a peu de problèmes car cela passe par les Directions Départementales des Services Vétérinaires qui sont très avertis du risque et conseillent efficacement les sujets.

\section{Vaccinations des professionnels de santé}

\section{Vaccinations obligatoires}

La liste détaillée des différentes professions de santé (et des étudiants et élèves des écoles de santé) concernées par les obligations vaccinales est rappelée dans le $B E H$ ( $n^{\circ} 29-30,2006$, voir [1]). Cette liste détaillée doit être prochainement complétée en particulier pour les indications de la vaccination anti-hépatite $B$.

\section{Diphtérie, tétanos, poliomyélite}

La vaccination est obligatoire. Les rappels doivent être réalisés tous les 10 ans avec une dose réduite d'anatoxine diphtérique

\section{Hépatite $B$}

Le schéma recommandé est un schéma classique de 3 injections. Si la primo-vaccination a été faite avant l'âge de 25 ans, on considère que le sujet est immunisé et qu'il n'a besoin ni de rappel ni de contrôle sérologique. Si la primo-vaccination a été faite après l'âge de 25 ans, on considère qu'un taux d'anticorps anti-HBs supérieur ou égal à $10 \mathrm{mUI} / \mathrm{ml}$ est un indicateur de protection [1]. Si l'on ne dispose pas d'un dosage des anticorps anti-HBs, même ancien, ou si le taux est inférieur à $10 \mathrm{mUI} / \mathrm{ml}$, on réalise une nouvelle injection vaccinale avec un contrôle du taux d'anticorps un à deux mois plus tard. S'il reste inférieur à $10 \mathrm{mUI} / \mathrm{ml}$, le médecin du travail décidera des modalités de rappel et de mesure des anticorps en fonction de l'exposition. Dans tous les cas, le nombre total d'injections, y compris les 3 injections de primo-vaccination ne doit pas dépasser 6 . Les modalités de contrôle doivent être reprécisées mais le nouveau texte réglementaire n'est pas paru. Le plus important est certainement de rappeler cette obligation vaccinale aux étudiants et élèves des différentes filières des professions de santé qui sont âgés de moins de 20 ou 25 ans. Enfin, le Conseil Supérieur d'Hygiène Publique a conseillé dans deux avis de 2003 pour les professions de santé les plus exposées (médecin, dentiste, infirmier et sage-femme, pharmacien-biologiste et laborantin-préleveur) d'abaisser l'âge de la primo-vaccination au-delà duquel un contrôle sérologique est nécessaire de 25 ans à 13 ans.

\section{Typhoïde}

Malgré le faible nombre de typhoïdes en France (moins de 200 cas par an), le vaccin reste obligatoire pour les personnels des laboratoires d'analyse biologiques (1 injection puis revaccination tous les 3 ans).

\section{Tuberculose}

Une IDR à la tuberculine (Tubertest $5 \mathrm{U}$ ) est obligatoire à l'embauche de même qu'une vaccination même ancienne par le BCG (preuve écrite ou cicatrice).

\section{Vaccinations recommandées \\ Coqueluche}

La vaccination contre la coqueluche est absolument indispensable avant le premier contact avec l'hôpital ou avec des collectivités d'enfant. En effet, avec l'extension de la couverture vaccinale chez les jeunes enfants, qui atteint 87 à $92 \%$ avant 5 ans [5], l'épidémiologie de la maladie s'est totalement transformée en France. Une coqueluche clinique, avec souvent des apnées graves, est diagnostiquée avant tout chez les enfants de moins de 6 mois. Ces enfants sont contaminés par leurs frères et sœurs plus âgés et surtout par leurs parents, immunisés dans l'enfance, qui, eux, font une coqueluche paucisymptomatique, limitée à une toux banale, mais responsable de la transmission du bacille dans la communauté [6-9]. Les recommandations récentes, possibles grâce au vaccin acellulaire, préconisent un rappel vers 10-12 ans, et surtout un rappel chez les jeunes adultes, avant la naissance de leur premier enfant (ou, pour la mère, juste après) et devraient entraîner une amélioration nette. Cependant, si la couverture vaccinale «coqueluche» s'améliore chez l'adolescent ( $55 \%$ à 15 ans), elle reste très faible chez le jeune adulte, en raison d'une diffusion insuffisante de l'information aux maternités et médecins généralistes. Ces coqueluches de l'adulte sont fréquentes dans les hôpitaux et plusieurs épidémies ont été observées récemment dans des services d'adultes. La situation la plus grave est celle où un membre du personnel soignant est atteint parce qu'il risque de contaminer de très jeunes enfants ou des adultes fragilisés (déficit immunitaire, personnes âgées, etc.). La recommandation officielle est de vacciner les membres du personnel médical et paramédical des maternités, des services de pédiatrie et de néonatologie. Cette recommandation est étendue, à juste titre, à tous les étudiants des filières médicales et paramédicales, en précisant que le rappel dTPolio (diphtérie faible valence-tétanos-polio) doit devenir un rappel dTPolioCa (coqueluche acellulaire).

II faut probablement aller plus loin. En effet, des médecins ayant un exercice exclusif auprès d'adultes peuvent être atteints et contaminer leur nourrisson, comme cela a été rapporté récemment à Paris [9]. II semble également 
raisonnable que les instituteurs en formation et le personnel des écoles aient, à l'occasion d'un rappel habituel par dTPolio, une injection de dTPolioCa. II n'existe malheureusement pas de vaccin avec une valence isolée de coqueluche acellulaire. Mais on sait maintenant que le rappel dTCaPolio peut être fait sans danger 2 ans après une injection de dTPolio aux adultes pour lesquels il est indiqué. En revanche, en dehors du rappel conseillé des adultes jeunes susceptibles d'avoir un enfant et qui concerne l'ensemble de la population, et est particulièrement utile pour le personnel de santé, il n'existe actuellement aucun argument pour conseiller un rappel plus tardif du vaccin anti-coquelucheux.

\section{Varicelle}

La vaccination contre la varicelle est recommandée chez les personnes sans antécédents connus de varicelle, ayant une sérologie négative, et susceptibles d'être en contact avec de jeunes enfants (personnels de crèche et collectivités d'enfants), et chez les professionnels de santé en particulier ceux qui exercent dans des services où les patients sont à risque de faire une varicelle grave (immunodéprimés, greffes, néonatologie, maternité, etc.). Les élèves et étudiants des professions de santé non immuns sont à risque, non seulement de transmettre une varicelle à des patients fragilisés, mais aussi de faire eux-mêmes une varicelle, toujours plus sévère chez l'adulte. Il est donc indispensable de connaître leurs antécédents et de vérifier leur sérologie en cas de doute. II peut y avoir des varicelles d'intensité modérée, dont la symptomatologie se limite à quelques boutons, et d'autres qui passent inaperçues, mais c'est assez rare. L'absence de souvenir de varicelle dans l'enfance est souvent associée à une sérologie négative. II faut également rechercher cette information chez les élèves enseignants et proposer une vaccination à ceux qui ne sont pas protégés. Parmi les patients atteints de varicelle sévère et hospitalisées chaque année en France, on trouve un nombre non négligeable d'instituteurs. L'attitude officielle va dans ce sens en précisant: «personnels de collectivités d'enfants ».

\section{Rougeole}

Les professionnels de santé en poste ou en formation, sans antécédents de rougeole et dont la sérologie est négative, et à risque d'être en contact avec des sujets atteints de rougeole doivent recevoir une injection de vaccin trivalent (rougeole-oreillons-rubéole).

\section{Grippe}

La vaccination concerne tous les professionnels de santé et toutes les professions en contact régulier avec des sujets à risque (personnes âgées ou avec une maladie chronique nécessitant la vaccination, personnes séjournant dans un établissement de long ou de moyen séjour) de façon à éviter de transmettre la grippe. De la même façon, de nombreux pédiatres recommandent la vaccination contre la grippe aux parents d'un prématuré avant que celui-ci ne regagne le domicile en automne ou en hiver. En cas de survenue de pandémie grippale, la vaccination antigrippale serait rendue obligatoire pour toutes les professions de santé.

Enfin, depuis 1999, la vaccination antigrippale est appliquée dans les armées selon un schéma triennal [2].

\section{Hépatite $\mathbf{A}$}

La recommandation pour la vaccination contre l'hépatite A concerne les sujets professionnellement à risque de contamination, c'est-à-dire les personnels des crèches, d'internats, d'établissements et de services pour l'enfance et la jeunesse handicapée. Mais il faut bien comprendre que tout le personnel de santé s'occupant d'enfants est à risque. En effet, l'hépatite $A$ est transmise par les enfants les plus jeunes qui restent souvent asymptomatiques [1013]. La France est un pays où il existe des flux migratoires saisonniers importants. De nombreuses familles passent les vacances avec leurs enfants en Afrique du Nord ou en Afrique sub-saharienne et il est fréquent que les enfants n'aient pas été vaccinés au départ car le vaccin est trop cher et n'est pas remboursé. Ils développent au retour une hépatite $A$ contractée sur place et la répandent dans la communauté. Les personnels de santé sont exposés de cette façon. II semble important de rappeler aux infirmières des services de pédiatrie et d'urgence, et aux étudiants en médecine et aux médecins, que le risque est loin d'être négligeable et que la vaccination est particulièrement utile même si cette vaccination ne fait pas l'objet de recommandation pour les professionnels de santé. À notre sens, elle doit être recommandée.

\section{Les vaccinations dans les armées}

Le calendrier vaccinal utilisé dans les armées est actuellement défini par une instruction ministérielle de février 2005 [2]. Le Service de Santé des Armées assume la responsabilité de l'exécution des vaccinations. L'objectif est une stratégie de protection à la fois collective et individuelle. Les militaires sont soumis au calendrier vaccinal habituel, et quelques vaccinations réglementaires ont été introduites.

La vaccination contre les méningites à méningocoques est effectuée depuis plusieurs années. Avec l'émergence de la souche W135 en Afrique et au Moyen-Orient, la réglementation a été légèrement modifiée et depuis le $1^{\text {er }}$ janvier 2005 , la vaccination est pratiquée lors de l'incorporation avec un vaccin anti-méningococcique tétravalent ACYW 135 avec une revaccination tous les 3 ans. La vaccination contre l'hépatite $B$ est réglementaire pour tous les militaires. Les professionnels de santé des armées doivent être immunisés contre l'hépatite $B$, conformément aux obligations réglementaires pour tous les professionnels de santé. Pour les militaires n'appartenant pas aux professions de santé, qui n'ont pas été antérieurement vaccinés, la vaccination contre I'hépatite $B$ consiste en l'administration de 3 doses de vaccins, deux à 1 mois d'intervalle et la troisième un an plus tard, sans contrôle sérologique. Le contrôle sérologique est seulement demandé pour les personnels à risque : gendarmes, pompiers, secouristes. 
La vaccination contre la typhoïde est réglementaire pour les militaires, avec une vaccination tous les 3 ans. La vaccination contre l'hépatite $A$ est également réglementaire chez tous les militaires, de même que la vaccination contre la fièvre jaune. Le rappel de la vaccination vis-à-vis de la fièvre jaune est prescrit uniquement pour le personnel désigné à servir Outre-mer et en opération extérieure.

Depuis 1999, la vaccination anti-grippale, selon un schéma triennal, est introduite dans le calendrier vaccinal des armées.

Enfin, selon l'endroit où les militaires doivent servir, d'autres vaccinations circonstancielles sont appliquées: contre l'encéphalite japonaise, l'encéphalite à tiques, la rage et enfin la leptospirose.

\section{Voyages et risques professionnels}

Les vaccinations recommandées aux voyageurs doivent être systématiquement envisagées pour les professionnels amenés à se déplacer dans le cadre de leurs activités professionnelles $[4,12]$. En effet, beaucoup de ces déplacements se décident peu de temps avant le départ, et il faut s'assurer que le sujet a été bien vacciné. Les séjours prolongés Outre-Mer se font souvent en famille et sont le plus souvent prévus à l'avance, les personnes sont averties et favorables aux vaccinations. Mais le plus important est de juger du risque sanitaire sur place. II n'y a aucune commune mesure entre le jeune étudiant qui part ponctuellement travailler pour une organisation non gouvernementale dans une bourgade angolaise ou d'Asie du sud-est et la famille expatriée qui va vivre dans une maison confortable en Afrique.

Un départ brutal peut avoir des conséquences dramatiques si les dispositions nécessaires ne sont pas prises. Aux États-Unis, des cas de décès par fièvre jaune touchant des ingénieurs qui ont dû faire un voyage éclair dans une région à risque en Amérique du Sud, sont régulièrement publiés. Ils n'ont pas été vaccinés avant le départ et la maladie est pratiquement toujours fatale [13]. L'Afrique est beaucoup plus endémique pour la fièvre jaune que l'Amérique Centrale et l'Amérique du Sud, et les contrôles fréquents de la vaccination anti-amarile dans les aéroports africains obligent à être correctement vacciné.

Mais en dehors des vaccinations qui peuvent paraître exotiques, le plus important est de s'assurer que le calendrier vaccinal «national » est correctement fait, avec les rappels nécessaires incluant diphtérie, tétanos et poliomyélite. II faut s'assurer par ailleurs que la vaccination anti-hépatite $B$ a été correctement pratiquée lorsque le séjour sera prolongé dans des régions de moyenne ou forte endémie pour l'hépatite $B$.
La vaccination contre l'hépatite $A$ est absolument indispensable car le risque est important dans la plupart des pays d'Outre-Mer, y compris ceux de la zone méditerranéenne, incluant les zones sud de l'Europe. II faut considérer qu'un sujet de moins de 30 ans non vacciné est à risque s'il part dans une zone d'endémie. Deux solutions s'offrent alors : soit contrôler la sérologie du virus de l'hépatite $A$, soit vacciner sans faire de sérologie.

Les autres vaccinations sont faites en fonction de la situation épidémiologique. La fièvre jaune est réalisée avec une injection au moins 10 jours avant le départ, et la validité est de 10 ans. Elle est possible dès l'âge de 6 mois. Les zones d'endémie amarile sont bien connues en Afrique sub-saharienne et en Amérique du Sud.

L'encéphalite japonaise est une vaccination disponible en ATU (autorisation temporaire d'utilisation) réservée aux Centres de Vaccinations Internationaux. Elle est indiquée (3 injections à J0, J7, J21) en cas de séjour prolongé en zone rurale, à la saison des pluies, en Asie du Sud et de l'Est.

La vaccination contre l'encéphalite à tiques n'est indiquée que pour un séjour printanier ou estival en zone rurale en Europe centrale et orientale. Elle est rarement indiquée pour des raisons professionnelles, et elle n'est pas recommandée actuellement sur le territoire français.

Enfin, le vaccin anti-méningococcique $A+C$ (une injection au moins 10 jours avant le départ) est indiqué pour un séjour prolongé en situation de forte promiscuité et en période épidémique en Afrique sahélienne, c'est-à-dire la saison sèche. Ce vaccin ne suffit plus maintenant pour le pèlerinage à la Mecque puisque la vaccination tétravalente ACYW135 est exigée par les autorités saoudiennes. Les armées, nous l'avons vu, utilisent également le vaccin tétravalent. La prédominance de W135 dans la zone sahélienne semble s'atténuer depuis 2 ans, mais beaucoup le conseillent encore. Il est utile de rappeler que seule la fréquentation étroite de collectivités d'enfants en saison sèche constitue un risque.

Enfin, le vaccin contre la typhoïde est encore souvent conseillé, mais son efficacité est discutée.

Quant à la vaccination contre la rage à titre préventif, elle n'est indiquée que dans la mesure où il s'agit d'un séjour à l'étranger, aventureux ou en situation isolée dans une zone d'endémie, c'est-à-dire essentiellement l'Asie du Sud-Est et l'Inde. En fait, il faut essayer de raisonner de façon pragmatique. II n'y a pas lieu de vacciner à titre préventif contre la rage un sujet qui peut avoir accès très rapidement au vaccin post-exposition et aux immunoglobulines dans la région où il va résider. II s'agit donc là que d'une prévention individuelle à discuter au cas par cas.

Enfin, deux recommandations sont particulières: le choléra et la grippe. Le vaccin contre le choléra, administré par voie orale, est rarement utilisé. Cependant, quelques $0 N G$, travaillant dans des zones d'infestation importante du choléra (Angola en particulier) le recommandent pour leurs membres. Quant à la vaccination antigrippale, elle est recommandée en fonction de la destination et de la saison. Les professionnels du tourisme doivent être vaccinés contre la grippe. Il est important également de rappeler que les saisons d'endémie sont inversées et que ce n'est pas toujours le même virus qui circule dans l'hémisphère sud et dans l'hémisphère 
nord. II faut donc essayer, dans l'hémisphère sud, de se vacciner au bon moment, et avec les souches indiquées pour la vaccination à la saison considérée. Ce vaccin est assez difficile à trouver en France, même en ATU, même dans les centres de vaccinations internationales, et souvent il faut se résoudre à se faire vacciner dans le pays de destination.

\section{En conclusion}

Le plus important est d'être pragmatique et d'évaluer exactement le risque qui est toujours double. Le sujet concerné peut-il contracter une maladie liée à son activité professionnelle? Le sujet peut-il transmettre une infection à des patients fragilisés dans son entourage? Cette évaluation n'est possible que si l'on dispose d'une information exacte sur les conditions de travail et les recommandations officielles. Le médecin du travail est indispensable, mais le médecin traitant joue un rôle majeur de conseil par la confiance que lui apporte le patient. Là encore, il faut rappeler que la vaccination est un acte de médecine individuelle au service de la communauté et passe toujours par le dialogue singulier médecin-malade. $\diamond$

\section{SUMMARY}

Vaccines and exposed occupations

The use of safe and efficacious vaccines in occupational settings to protect workers from diseases to which they may be exposed is obvious and has been included in the employment law. Healthcare workers are particulary exposed. Immunization has two purposes: protect the worker from contracting a disease, but also prevent him from disseminating the disease to weakened patients. It is important not only to take into account existing recommendations for immunization, but also to envisage their extension to teachers and staff of nurseries and primary schools. Routine vaccination against whooping cough, varicella, measles and hepatitis $A$ is particularly warranted in these categories. Recommandations should also extend to medical students who are too often poorly protected and insufficiently warned against potential occupational exposure to pathogens and dissemination to their patients. $\diamond$

\section{RÉFÉRENCES}

1. Calendrier vaccinal 2006 et Avis du Conseil Supérieur d'Hygiène publique de France. BEH 2006; 29-30: 212-24.

2. Direction générale de la Santé. Comité technique des vaccinations. Guide des vaccinations (édition 2006). Collection Varia. Saint Denis : Inpes, 2006. http://www.sante.gouv.fr/ (rubrique vaccinations).

3. Guide canadien d'immunisation, 6 ed, 2002. http://www.phac-aspc.gc.ca/

4. Santé des voyageurs et recommandations sanitaires 2006. BEH 2006 ; 23-24: 153-64.

5. Couverture vaccinale des enfants et des adolescents en France. BEH $2007 ; 6: 45-9$.

6. Von Konig CH, Halperin S, Riffelmann M, Guiso N. Pertussis of adults and infants. Lancet Infect Dis $2002 ; 2$ : 744-50.

7. Edwards KM. Overview of pertussis: focus on epidemiology, sources of infection, and long term protection after infant vaccination. Pediatr Infect Dis J $2005 ; 24$ : S104-8.

8. Gilberg S, Njamkepo E, Du Chatelet IP, et al. Evidence of Bordetella pertussis infection in adults presenting with persistent cough in a French area with very high whole-cell vaccine coverage. J Infect Dis 2002; $186: 415-8$.

9. Raymond J, Armengaud JB, Cosnes-Lambe C, et al. Pertussis in young infants: apnoea and intra-familial infection. Clin Microbiol Infect 2007 ; $13: 172-5$

10. Gendrel D. Vaccination contre l'hépatite A chez l'enfant. Arch Pediatr $2004 ; 11: 1360-6$.

11. Wasley AM, Samandari T, Bell BP. Incidence of hepatitis A in the United States in the era of vaccination. JAMA $2005 ; 294: 194-201$

12. Craig AS, Schaffner W. Clinical practice : prevention of hepatitis $A$ with the hepatitis A vaccine. $N$ Engl J Med 2004 ; $350: 476-81$.

13. OMS. Voyages internationaux et santé. Vaccinations exigées et conseils d'hygiène. Guide pratique. Genève : OMS, 2005. http://www.who.int/ith/fr/

14. OMS. Situation de la fièvre jaune en 2004, Afrique et Amérique du Sud. Releve Epidemiol Hebd $2005 ; 29$ : 250-6.

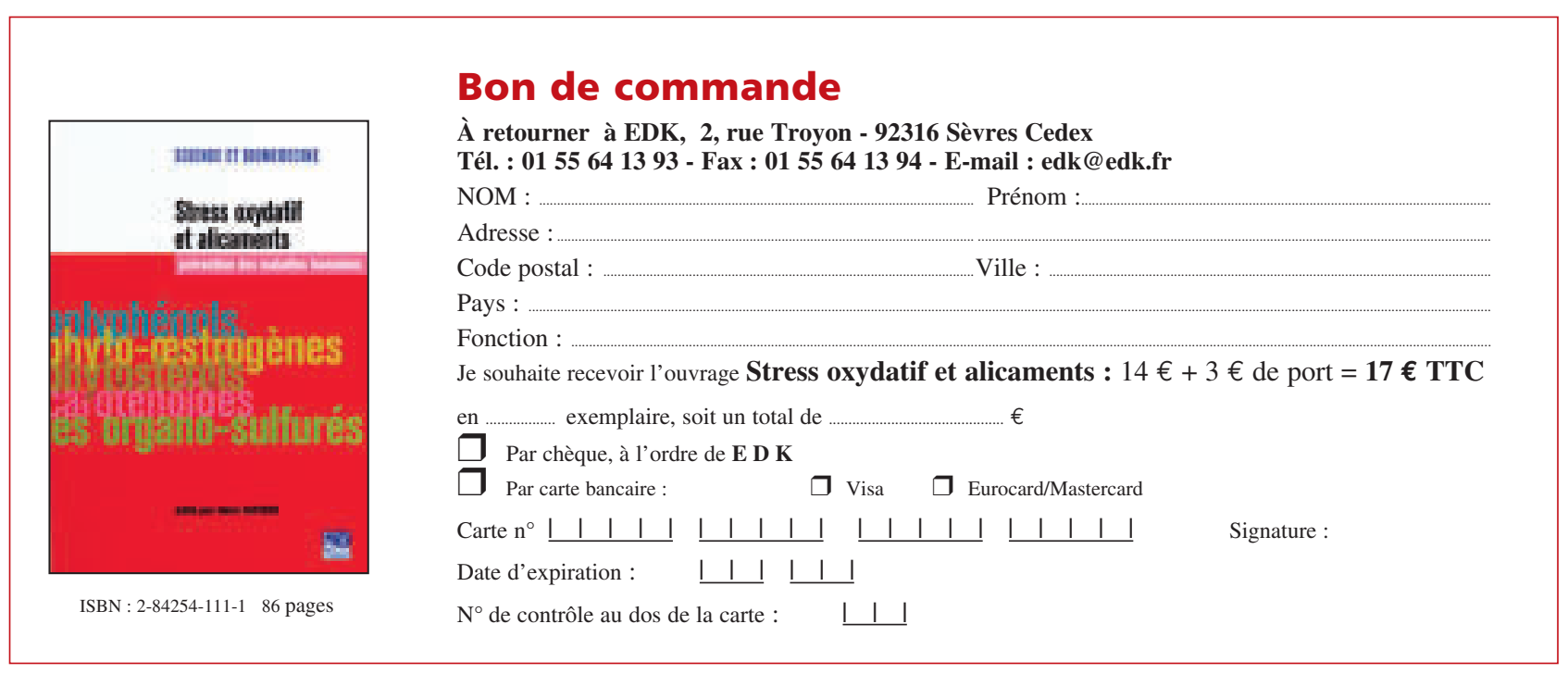

